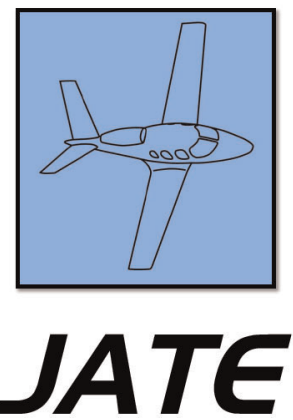

Journal of Aviation Technology and Engineering 10:1 (2021) 12-20

\title{
Changing the Process in Educational Field and Air Navigation through Advances in Hologram Technology
}

\author{
Camilo Fernandez Sr.
}

Universidad de Antioquia

\begin{abstract}
The objective of this piece is to propose reasons that change the way air traffic controllers and others learn and work in air navigation, but also how they can improve workloads, due to the implementation of holographic radar. This research also aims to describe how this could reduce the cognitive load of an operator, thanks to the improvement of visual perspective and capacity of analysis, in order to more easily control an aircraft.

Seeing that the visual facilitation is well known, radar is able to represent in three dimensions and in detail that which could not previously be perceived. This makes a substantial difference in the comfort and ease of training new controllers. In conjunction with operators, the objective of this technology is to save both hours and cost of training. This is of tremendous benefit, as some countries spend millions of dollars trying to educate the personnel in charge of air traffic control. In addition, a goal of this technology is the further improvement in air safety in a sector that demands more jobs and more availability to people in the industry, with an increasing number of aircraft in the world. This makes air traffic control one of the more demanding careers in the global arena.
\end{abstract}

Keywords: aerial navigation, holograms, cognitive load, learning, radar

\section{Introduction}

Since the invention of the airplane, mankind has been able to shorten distance across the globe. Journeys that used to take days, weeks, or months now require a few hours to travel from one place on Earth to another. The world is much smaller than it used to be, thanks to the creation of a particular machine that today is part of daily life, taking users where they want and reducing barriers between people and different places around the world.

Nonetheless, as the use of this technological innovation increases - caused by high demand of passengers, low prices of travel tickets, and the globalization in business and tourism - this enables new routes for passengers and flights to different zones. The number of incidents, resulting in possible accidents, has increased considerably, putting the industry and its workers at risk, as well as lowering the confidence of travelers using airplanes. This also increases the workload for pilots, technicians, and obviously air traffic controllers, as employee tasks have been intensified. Most of these workers have to be available seven days a week, 24 hours a day, and have little rest time to keep them capable of controlling the sky. It is, therefore, vital that new technologies be made available that can ensure air safety and gain the confidence of the industry. 
Holographic aeronautical radar is a new technology which will help operators to improve their work and reduce training time, making the control of airports, airspace, and routes safer. This innovation changes the way controllers and the aviation industry see the idea of airplanes in a simulated, virtual, three-dimensional (3D) space. This helps to verify where aircraft are located in a simulated area, in real time. Giving animations and model figures in $3 \mathrm{D}$, in a virtual environment, facilitates the understanding of what is seen, and therefore reduces the mental effort to learn and imagine where objects are and organize them in the space of two dimensions, in which operators learn and work currently.

The work of an air controller, or rather radar operator, is one of the most stressful in the world. It also requires years of training and constant hiring, in addition to specific skills and qualities. The radar operator suffers constant mental stress due to job risks: the candidate and even the current employers must engage in psychological interviews during their employment, and together they must work under pressure and are responsible for hundreds of lives. An error on their part can cost not only their work, but the reputation of the industry. Since the dawn of the aviation industry, human error has been one of the most critical factors that can lead to accidents. Radar technology emerged and changed aerial navigation, making the work of air traffic controllers easier. As passengers embraced this new technology, more and more accepted the plane as a means to arrive more quickly at the destination of their dreams.

This is one of the most significant advances in holographic technology that currently exists, although there are some more surprising advances with respect to this innovative science. Those that can be used to build prototypes, which is part of this writing, also in the section of related works, I have mentioned recent investigations related to this idea. They do not add to the purpose that this paper is discussing, since it is a novel proposal that has an object to be taken into account. Perhaps for future events, with adequate resources, they can build devices that show these characteristics.

\section{How 3D Perspective Changes the Way the World is Seen}

Human beings are different from other animals in their increased brain capacity, and, in conjunction with their visual acuity, humans are able to make and improve tools for manufacturing, and construct civilizations. This is possible thanks to the visual perspective that mankind has to not only build tools, but also to develop structures and create communities where everyone plays a vital role for the progress of humanity.

From the beginning, humans have expressed their ideas via constructions and messages, and in some cases these have withstood the test of time. An example of this is cave paintings in some European caverns and Egyptian hieroglyphs in pyramids. Accompanying human use of reason was the desire to capture ideas and preserve them. Despite this, some facts are difficult to comprehend, because the language in which they were written no longer exists or is not spoken by modern humans. A clear example of this is the so-called Rosetta Stone (Downs, 2006). It required 18 years to translate, as it was written in three different languages, including Egyptian hieroglyphs (Adkins \& Adkins, 2001). To know their meanings, it would take decades to translate, as there are images and symbols of past cultures. The essence of their ideas is not clear yet, because there are many unknowns about their primitive language.

Following the invention of writing and books, many ideas have remained due to the preservation of copious amounts of human knowledge. Nowadays, this format can be seen in different media, not only in the form of letters but also in printed images, video, and audio. In ancient times, people had to draw for hours to show a concept. With today's new technologies like printed media, a publisher and even an amateur can make presentations in a matter of minutes. Despite this, there is so much content that most of it is unable to be interpreted as it is pervasive and complex to read. Many modern databases are textbased, flat images posted on sheets of paper. This material has been a fundamental axis in the transmission of messages in humanity, accrediting people to read ideas, not only authors, but also information on concepts that are hard to explain with words. Although this has allowed people to have access to specific data permanently and efficiently, there are cases in which those concepts are complicated. Likewise, there are people who have little cognitive ability, or can distinguish things better by other means.

That is when the internet, and the high apogee of portals like YouTube and Facebook-where internet surfers can observe all kinds of topics-is capable of displaying generating materials in other ways, so that ideas or universal objects can reach people more easily, no matter how difficult it is for a person to read a book. Whether it is text or looking at an image, what humans have in common is that they discern ideas more quickly if they come in the form of animated and narrated images, which is known as the principle of modality and redundancy. This principle is so valid that it has been used in projects for people with problems corresponding due to dyslexia (Knoop-van Campen et al., 2018), and it is here that new technologies, similar to holograms, have arrived to establish a new means with which ideas can not only remain, but can be issued in such a way that, without regard to complexity, they can reach a person effectively, knowing that this person is not able to read, or that they have difficulties in their learning.

It is here when the images that were previously static and could only be watched from an angle become images with relief that show the user their quality, and we can be undoubtedly aware of what idea is said or shown. This can 
be observed through virtual reality applications, which allow a user to see objects or things virtually, thanks to mobile apps or special lenses, that can see abstractions that are difficult to imagine with words. If one takes for an example, if it is a cell or a black hole, it is explained with words or in a text. These are abstractions challenging to imagine for an ordinary mind, all because these objects, although they exist in real life, cannot be seen with the naked eye. A cell is minimal and microscopic in size, while a black hole exists in distant places of the universe, so they are unable to be easily seen. Instead, through showing them in 3D imagery, showing them in a virtual representation of how they exist in real life, it allows people to remember something that previously would have been more complex to imagine.

That is why pedagogues, creators of content, and companies have bet on more and more use of formats in 3D. This is mainly through virtual reality, digital applications, and animations, which permit future internet users to see simulated things before buying them, or play and visualize dynamic and deducible learning contents. 3D has arrived as a way to not only to entertain, but also to show usefulness to the people who need it, because by other means they would not be able to retain the evidence in an efficient way. This is where holographic technology has come to stay, and together with virtual reality, be the new way of communicating the different thoughts of individuals in a bright and striking way.

\section{Common Applications of Holograms}

Although the technology to project holograms is still in the early stages, and although development is limited to static images in $3 \mathrm{D}$, its realism is undoubted. There are similar developments that show interactive animated images in the third dimension, with unlimited content for all types of people; however, according to the real concept of holograms, they should be an image that is projected in the air without any assistance, as if floating. What exists today requires reflective surfaces, lenses, or virtual reality viewers to be able to observe the images and interact with them. There are also approximations of projectors that show pictures in the air. With some help, with some improvement, it is only a matter of time before holograms will project as if they were a television.

Companies and individuals have dedicated efforts to create content for this type of technology, particularly in the educational, commercial, and entertainment fields. They have applied basic notions comparable to Pepper's ghost reflexes (Costa, 2014) to show products, make explanatory lessons, together with virtual companions. The hologram visualization is based on reflection through a surfaces to show content, and the people involved in this field of innovation, at the moment use some basic animation for comercial use, in a near future there are people in charge to develop more content like educational lessons and animated stories to be captivated in a holographic interactive projection.

Holograms have no imaginary limits to be shown graphically. Their quality of visualization makes them a tool, by way of projecting images, thus captivating the eye of people. This is possible by the method of modality and redundancy (Ivashov et al., 2017), which makes people pay more attention to issues if they come with animated movement and narrations, also adding interactivity. It is irrelevant whether it comes static and two-dimensional; video games use this reference a lot-only they are not broadcast outside of televisions. With holograms it is intended to change this trend, so that the content can reach more people, but this metadata can be viewed more enjoyably, depending on the intended audience.

Holographic displays serve to show things that are impossible to be seen in reality or are difficult to see at a glance. In the case of medicine, the human body is unable to be seen except through radiographs or electronic scans. When a person needs an operation, with holograms doctors can see a real representation of the human body without intervening and, therefore, can make a more detailed analysis of what procedure should be done without causing more significant harm to the patient. Medical students can also be taught how to do procedures or view how the human body parts work through this method. Additionally, in the field of navigation, a pilot or driver can have holographic optical elements that are represented on a display in the windshield that indicate where the road or runway is in adverse conditions. A radar operator also could have a device with these capabilities to be able to see any additional requirements regarding the control of airplanes in the air.

Finally, the educational component is where holograms have the most enormous potential because the arenas in which 3D animated images can be utilized which represent universal ideas are varied. They can be used in all primary subjects, both mathematics and physics, and natural sciences. The processes of learning, in turn, are faster because the mind can better cognitively apprehend interactive images and narratives which demonstrate what exists in the real world better than through letters and static words. It has already been shown with television, the audience for which far exceeds that of the press and radio. Video web portals are already becoming more popular than these forms of media, as the public who accesses them spend many hours of the day watching informative and entertaining content. With holograms, a new form will be created to see content of any kind, not only on the web, but also in the real world.

\section{How a Hologram Helps an Individual's Information Retention}

It has been discussed how the principle of modality and redundancy could make the difference between information 
which is not able to be easily remembered and other that can, if permanently retained. Holograms have the faculty by their graphic characteristics to enter the brain of a person with ease. It is more comfortable when a person with visual learning tendencies uses this type of tool. Additionally, the principle of modality and redundancy establishes that it helps people with cognitive difficulties to improve their learning (Smith \& Ayres, 2016). Significant research has seen in this technology a basis to facilitate the understanding of interactive thematic material. This postulate mentions that it is a procedure which performs a cognitive process. According to recent data, it changes the thinking structure of the individual. The person learns the concepts, and from this, makes a comparison and analysis to verify if the records obtain a kind of "reconciliation," which confirms if it is useful, and whether there is a broad reorganization of knowledge (Barriga \& Hernández, 1999).

According to Barriga and Hernández (1999), the methodology of meaningful learning dictates a series of conditions for the process to be carried out successfully. This consists of material types or contents presented in a certain way to the previous knowledge of the individual. There must also be an attitude and willingness to learn on the part of the person, and an adequate guide of the content creator. This is why slogans must have a structure where it admits the ease of acquisition of information, and new knowledge of part of the content creators, towards the people who will visualize them.

It is well known that people learn in a better and more accurate way through a combination of words and images. The famous phrase "a picture is worth a thousand words" indicates if an image expresses events in a person's mind, it represents more easily than a thousand written words. Bhatti et al. (2017) tried to illustrate the above through a study of the improvement of cognitive theory based on multimedia learning. Their argument focuses on the aspect of training, focused on the student who applies the human cognitive system, and how it affects their natural behavior, from which an emphasis is made where a human being can gain knowledge in a simulated environment. This is in addition to that acquired knowledge applied in real scenarios.

Bhatti's work on multimedia learning is based on three primary objectives which first define strengthening response - this is about the reactions of the learners that are tested and strengthened through a system of exercises and practice. Then there is the acquisition of informationwhich is multimedia tools and technology transferring material and text data to the learner. Finally, we have the construction of knowledge- - this is about the sense of logic and knowledge of a given subject in order to increase the retention of a given instruction.

Later, Bhatti mentions the process of using animation with cognitive multimedia learning, from which three derived principles are used. First is the belief of animationthis is about students expecting to interpret a topic quickly and with profound precision from a certain topic, in this case is an interactive multimedia theme. If the taught subject is created under the principles of 3D animation, along with text and voice narrations, create a cognitive process inside his brain. Then, we have the faith of temporal contiguity - this is derived from the basic rule where students perceive and learn a specific subject more profoundly and efficiently when the multimedia instructions contain text, words, or narration that is presented synchronically with the animation. Finally, there is the principle of personalization-which is based on that students learn more efficiently and deeply when it comes from an animation and narration if the latter comes in the form of casual conversation instead of an official technical format of words.

These principles simply rely on the theoretical validation that students are better able to focus and follow when narrations, textual caption, and animation are all synchronized together seamlessly. As a conclusion, multimedia learning is a technique that improves and facilitates the student's internal information system, which includes visual and verbal channels of how to process content. Furthermore, animation is a compelling medium and form of communication, so the knowledge of deep and complicated phenomena can be easily taught to students. It is evident that holographic visuals, which contain multimedia resources, are an excellent tool to provide the cognitive treatment that students and people in general can use to learn concepts quickly no matter how difficult they are.

\subsection{The Air Traffic Controller Cognitive Process and How Holographic Content Can Help to Improve It}

Air traffic controllers are the people who are in charge of controlling air traffic that is monitored by radar. This means that the airspace in which airplanes circulate is organized, and in this way accidents can be avoided. If two aircraft collide, depending on the location, the consequences of the crash can be catastrophic. The crew members, in addition to the passengers of the plane can die, and if the jets fall in a populated area, the people there may suffer the same fate as the passengers who boarded those flights.

Although it is a well-paid job, it has a very excessive workload. The reason for this is, if we mention the example of the air traffic controllers of the Federal Aviation Administration of the United States (FAA), they are among the best in the world. This is due to a very large volume of airplanes from different parts of the world landing in the USA. The number of both foreign and domestic US flights is vast and it is expected to rise with the reduction of the price of tickets and facilities to travel. Passenger demand increases, and therefore the number of airplanes circulating in the air and landing at airports will rise. For this reason, air traffic controllers that are currently in the FAA face an excessive workload. To become an air traffic controller with the FAA, one must be under 31 years old and have 
gone through three to four years of training. The FAA spends millions of dollars training and hiring new job positions. The recruitment process is very thorough, and the number of personnel hired to counter the high demand for aircraft is small. Today, controllers ready to retire and to enjoy their pension have had to defer their retirement time due to the fact that there are few radar operators with vast experience that face the challenges of a high demand of airplanes that circulate in the North American skies.

Air traffic control is one of the most mentally stressful careers in the world. Ae radar operator must not only organize in real time the aircraft located in a specific space, but also calculate how fast the planes are going according to their classification and the height of them by reading the parameters indicated on the screen. This has created a mental load that not even a day of six hours is enough for the mind to rest. A short but exhausting day due to the pressure that is predisposed, because if there is a fatal error, many people may die and put the credibility of the industry at risk.

The cognitive ability of the controller is limited because the information provided by the radar screen implies making extra effort calculating how to organize the aircraft and take them to the respective landing sites or aerial roads. Most monitors in control centers show graphics from a twodimensional point of view, which allow one to deduce heights and other parameters through indication numbers on the display, but is incapable of showing visually what plane it is, only through its number plate. The controller really only sees a green icon on the screen. Also, in certain areas with more traffic in the world, these operators must deal with up to 40 flights at a time and organize them in a parallel way so they will not collide, making quick mental calculations, a brain task that over the years diminishes cognitive capacity. It is for this reason that air traffic controllers must retire at an early age, due to the mental exhaustion caused by this task. This is a process that is costly to the industry. Hence, new technology is needed that will facilitate controllers' work and generate less mental load when viewing the inputs.

Software and a display that provides 3D figures in high relief grant the human eye the ability to collect evidence easily. Thus, the brain receives input effectively and can learn almost instantaneously. The binocular vision of humans gives the ability to capture detail better when it has depth. This is due to the object being related to something of everyday life, and the conceptual relationship of association is easier to assimilate. This can be defined as a kind of significant learning, which allows the individual to have clarity of the idea that is being seen, and the same comes with movement and interaction, the association of information with respect to the world around, which makes it easier to remember and learn.

A controller seeing airplanes virtually in a simulated space with depth and interactivity would result in the person being able to locate them more easily and organize them as if they were in real space. It is not necessary to imagine it by reason, if it is possible to see a representation of the real world that is known and can be easily associated, avowing in this process to do tasks faster and without too much mental load. Therefore, cognitive work is reduced, as the program is more easily visualized, making it actionable and memorable in time. In this way, the figures in 3D projected in the form of holograms will make changes in the way of visualizing substance, with the purpose of reducing training times. This then goes on to reduce the cost of training, facilitate the understanding of the surrounding airspace, and improve flight safety, considering it is easier to visualize objects in a virtual simulated environment than on a two-dimensional monochromatic screen.

It has been established that cognitive learning through multimedia technology is reinforced and leads to greater retentiveness. In the case of people who operate airborne radars in different parts of the world, the cognitive process works in the same way. Seeing that a holographic radar has multimedia content will encourage users to appreciate more deeply that they are human beings with a similar type of learning. They must be people whose characteristics mean they gain from visual stimuli-visual learners. The holographic technology will allow them to assume a more appropriate and fast way of learning with this method of work, processes such as the principle of animation, temporal contiguity, personalization, and finally modality and redundancy. This will mean people who come to train in this area are able to do so in a shorter period of time and then can do their work more effectively and safely, without considerable mental effort and can work for long times without cognitive fatigue.

\section{Current Holographic Technology that Allows 3D Radar Creation}

Although there are already programs that enable modelling in the third dimension of structures and objects, such as mountains, buildings, and air and land vehicles, the visual signal of them improving the 3D way is so they can eventually see without any type of parallax barrier. That is, without the help of special lenses or glasses that shows the image, as some technologies do; however, there are already devices that show real holographic features, although with some technical limitations. They stand out because they are very close to what is a conventional holographic gesture. There are two companies as examples for this case which show advances in the way of projecting 3D images in the air, and in the future. Their devices would serve for the construction of a holographic radar with the necessary characteristics of a reduction of cognitive load, ease of use, ease of projecting data, and so forth.

First, Japanese company Asukanet (2017), which manufactures the aerial imaging plate (AIP) called aska 
$3 \mathrm{D}$, leads the way in this type of motion, where the visual result is a reflection of a projected image from an inclined monitor positioned below it. The resulting image is a graphic floating in the air, and in fact is the only technology that can achieve this without any kind of visual aid. One of the drawbacks of this technology, however, is the field of vision reducing the direct perception of a person, which indicates that the floating images are only seen from one perspective and are unable to be viewed from other fronts. This means that the image is only seen if an individual stays in front of the plate that projects this visual: if the viewer relocates behind, or to the side, there is no possibility of being able to see the images. This technology is perfect for ATMs and security keys, because of the inability to see images unless one is standing directly in front. For radar, it is very useful to show 3D models in the air accessed only by the operator, who could see in the front and when viewing the 3D images for the air traffic radar controller it is easier to visualize them and also generates a cognitive-type rest which can be used efficiently. They would also enable more efficient control of aircraft traffic in real time, as if such traffic were in a simulated space. Previously designed software that simulates it shows graphics on a monitor below the plate, and the AIP is responsible for showing it in holographic form. In Figure 1 this type of technology is illustrated.

Apart from this technology, there is what is called volumetric display. There are several companies that work on and even offer this holographic technology. This can be referred to as holographic illusion. One company that stands out for its efficient way of showing information is Voxon Photonics (2017). It has a type of volumetric display that shows 3D figures that can be seen from different angles, and are projected in holographic form. This technology is different from that of Asukanet (2017) in that it uses a transparent bubble or cube inside of which the formed images are shown. It is a parallax barrier, but its objective is to contain the material that creates the pictures, playing games in it like chess. This product would be beneficial for the design of radar, because it can show maps and structures in a virtual way. Depending on the airspace that is going to be controlled, one can then design a larger device according to the requirements of the airport or the air traffic control station. It has the advantage that the software that it projects the objects onto-once the device receives the information, or the data to be plotted - can be observed in all possible locations looking out of the bubble. Figure 2 shows a demonstration of how to project the images in this kind of technological innovation.

From all of the above, it is now possible to create a tool that improves the way to see an airspace. This is with the aim of assimilating it in a better way, and being able to adapt to aircraft more quickly, not only visually but mentally, through an animated $3 \mathrm{D}$ visualization, which is a scale representation of the real environment. This enables a person to see in the simulation how a plane moves in reality. The improvement of the visual perspective facilitates not having to make additional efforts or quick mental calculations, but to be able to organize the aircraft. The multimedia resources of the holographic radar will help an individual to discern in a more adequate way and without getting tired at the level of retentive processes of material and mental-cognitive effort.

\subsection{Architectural Radar Components with Holographic Visual}

Analyzing the principles which involve radio navigation in the air, a pilot can determine the position of an aircraft by triangulating it using two radio aids to locate stations. By means of the automatic directional finder or VHF omnidirectional radio range (VOR), controls located in the

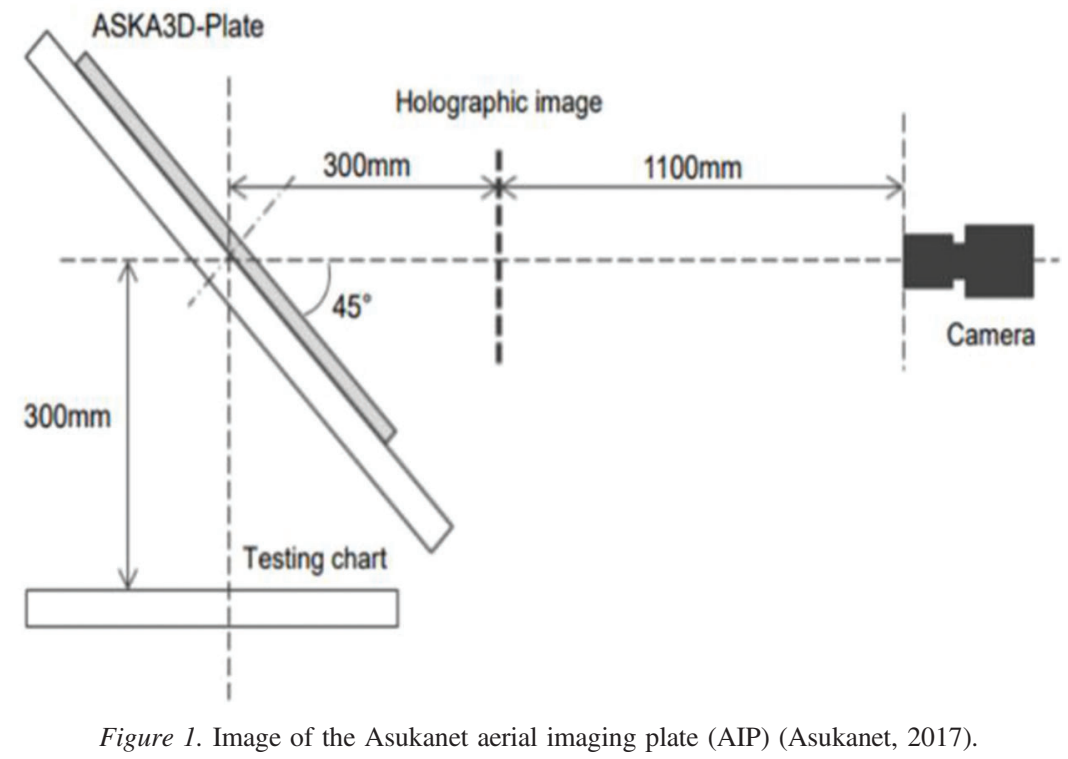




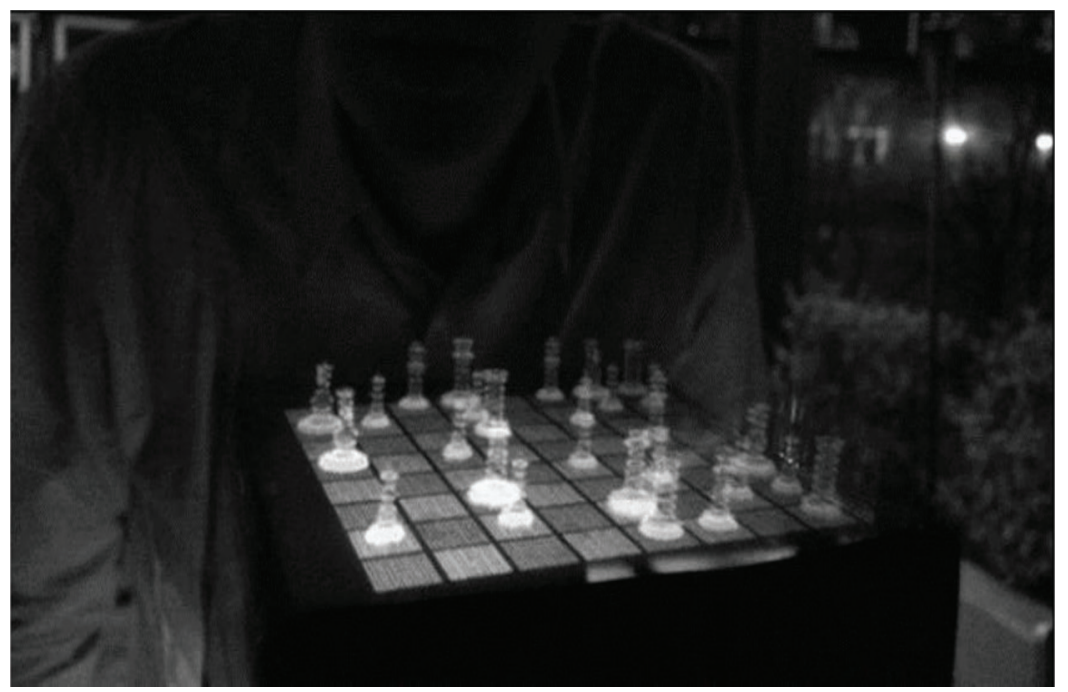

Figure 2. Voxon holographic device image demonstration (Voxon Photonics, 2017).

cockpit, all of these find the antennas that allow the plane to fly its course. Also, by polar coordination using the VOR antenna, shows as a results on the monitor the aircraft trajectory, measuring distances like attitude and airspeed, this information allows the controller to know exactly where the machine is. Although this method is active, it is not an optimal solution in areas subject to high traffic where the controller must be continuously informed of the exact position of all aircraft, at all times, within the controlled airspace. For this reason, the air traffic control center uses a radar monitoring system on the ground to automatically monitor the location of all aircraft within the control area, thus avoiding saturating the radio communication channels. With the information given by the radar, displayed at all times, the controller can make appropriate decisions regarding the delivery of the aircraft to the control center approach or departure. Furthermore, aircraft vectorization to avoid collision courses, maintain a safe separation altitude and distance between airplanes, along with locating and directing aircraft loss in their trajectory.

The air traffic control radar system on the ground consists of a primary surveillance radar (PSR) and a secondary surveillance radar (SSR). The PSR locates and tracks the planes within the control area by transmitting an energy beam reflected from the aircraft and returning to the PSR antenna. The SSR transmits interrogation signals to the transponder installed in the plane. Upon receiving the query, the transponder sends a coded response signal to the SSR system. Data collected from both PSR and SSR are used together to develop the visualization of the traffic situation in the controller radar range. This allows the controller to safely identify the aircraft equipped with transponders and to determine the scope and direction of all aircraft within the control area in order to organize them.

A primary radar system has several components. The first is the transmitter, which creates the radio waves sent and modulated, to form the train of detection pulses. The antenna must also amplify the signal to a high power level to provide an adequate range. Next is the receiver, which is sensitive to the frequency range that is being transmitted and provides amplification of the returned signal. Then there is the power supply, which provides electrical power to all the components. The largest consumer of energy is the transmitter, which may require several kilowatts of average power. The radar has a duplexer which is an alternate switch between the transmitter and the receiver of the antenna. Its purpose is to protect the receiver from the high-power output of the wire. During transmission of an outgoing detection pulse, the duplexer aligns with the transmitter for the transmission pulse duration. After this, the duplexer aligns the antenna with the receiver. When it sends the next pulse, the duplexer returns to the transmitter. A duplexer is not required if the transmitted power is low.

The final component, and the most important, is the antenna, which takes the pulse of the transmitter's radar and sends it to the monitored airspace. The antenna focuses energy in a well-defined beam, increasing the power and endorsing a determination of the direction of the target. The antenna must keep a record of its orientation, which can be achieved by a synchronized transmitter. There are also antenna systems that do not move physically, but are directed by electronic means (in these cases, it is known in which direction the beam orientation goes).

All of this detailed information is then displayed on a screen where the operator can observe the circulating airplanes. That is where the required changes will be made, so that the readout provided on the screen is holographic. In order for this to take place, there must be a server with the 3D modeling database of the terrain where the airspace is located and the airplanes flying over it. All this descriptive information must be stored previously and updated whenever there is a new aircraft 3D model flying. The 


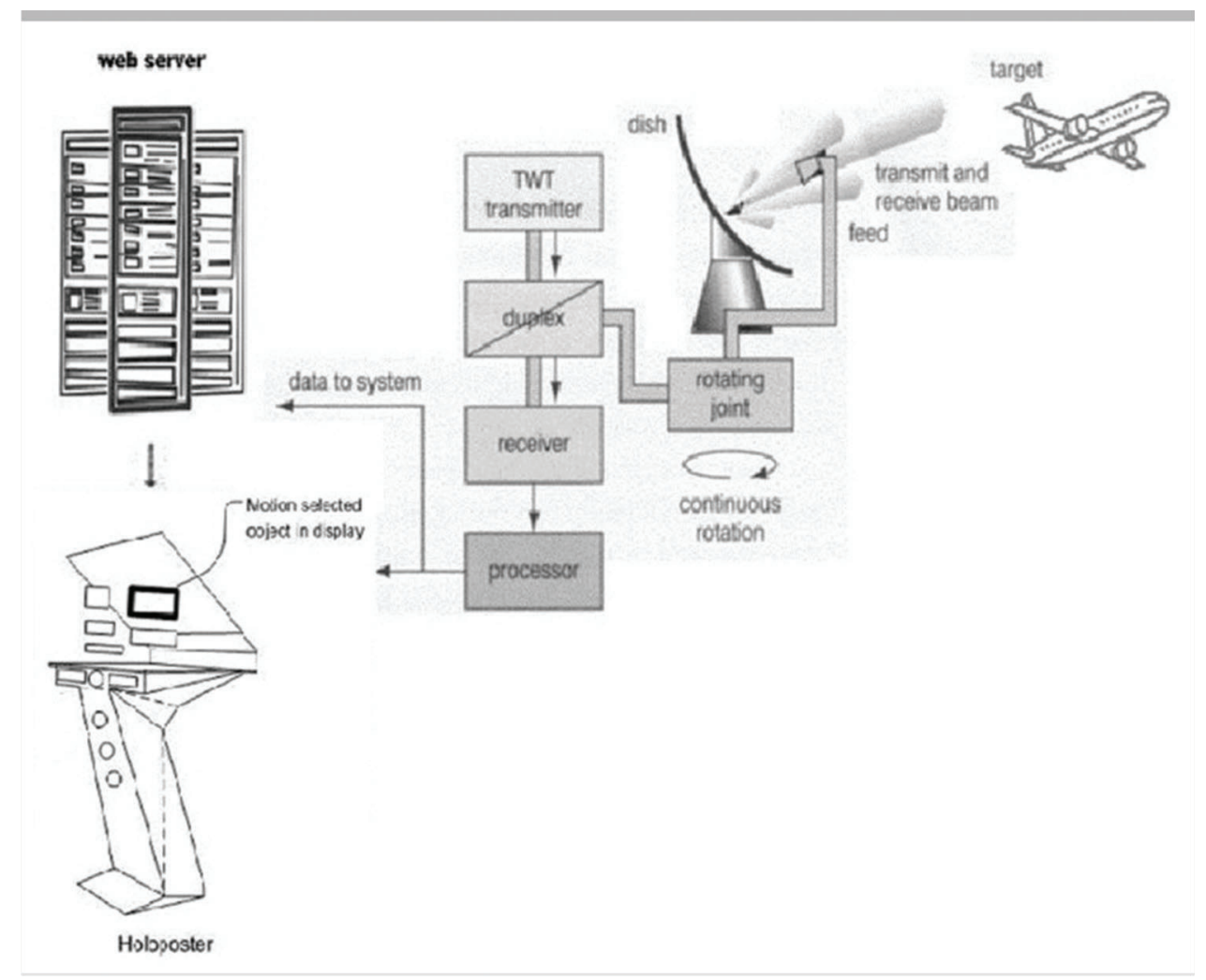

Figure 3. Holographic radar display modules (own elaboration).

terrain will not have to be modified except when there is a new obstacle that interferes with the normal air navigation. The server must be synchronized with the antenna and the holographic screen can have connection to reports coming from the web, in the event that one wants to connect with another air control server from another area remotely or for data analysis of any kind. In a synchronized form, the server supplies the graphics in real time to the display. Once the antenna data are received in this way, the visual representation in a 3D shape is seen, and the operator can interact with the format that is being viewed, dynamically and interactively, reducing the mental effort to know the types of aircraft that are being controlled, and the data they have as speed and height in real simulation. Normally, the radar controller sees items like direction, speed, and height, but they are numerical indicators that are observed on a flat screen, unlike the holographic display that shows the corresponding height of each aircraft in a simulated 3D environment. It is a copy of the real world transferred to the screen from the server that receives antenna data. Additionally, the message provided by the plane is because of the fact that the transponder must also have a modification in its software to contain additional data, which can be seen in the holographic screen, allowing the controller to direct the aircraft with the least possible effort. In addition, one can know which aircraft have priority, which can leave in time for better organization of traffic, and finally which are violating airspace or making unauthorized maneuvers or have any technical problem that the controller may notice. Figure 3 shows a model of the modules of a radar soon to be built.

\section{Conclusion}

It can be said that holographic technology, apart from having practical applications in the sense of offering products in an innovative and striking way, is a vital tool for the new educational methodology of cognitive learning. It can be used through multimedia resources, at an industrial level to improve the work method of people working in sectors that involve high accident risks, therefore reducing their workload and jointly their training and training time. This new technology will favor improving the quality of life of those who use it, and secure the skies and the devices that transmit data in a dynamic, interactive, and effective way.

\section{Related works}

Although holograms have evolved technologically and have made significant advances in recent years, their use has been more for commercial and entertainment applications. Industrial use is not ready yet, so this work is something new compared with others of the same relevance. Some reports give approaches about possible actions that assimilate to what is worked on in this writing, 
apart from the aforementioned references such as the work of Ivashov et al. (2002) who speak about holographic radars in the handling of electromagnetic pulses, but not of 3D viewing of visual objects. Ivashov et al. (2017) and other academics perform a similar job in which they describe more specific and concrete applications of the use of this technology. Uddman and Norberg (2017) refer to a position which realizes the use of a radar to find buried objects. All of this is an approximate relation with regard to the work in this report. The closest thing that resembles what has been mentioned here is the context observed with special lenses (Smith \& Ayres, 2016) where the objective is to achieve a technology that releases the assistance of the radar operator without the need for visual aids (Karthika et al., 2017).

\section{References}

Adkins, L., \& Adkins, R. (2001). The keys of Egypt: The obsession to decipher Egyptian hieroglyphs. New York: HarperCollins.

Asukanet. (2017). ASKA 3D-488 delivery specification sheet. Japan: Asukanet Company. Retrieved January 15, 2019, from https://aska3d. com/en/pdf/aska3d-488_delivery_specification.pdf

Barriga, D., \& Hernández, G. (1999). Estrategias para el aprendizaje significativo, fundamentos adquisición y modelos. México: McGraw Hill.
Bhatti, Z., Waheed, A., Asghar, G., \& Hussain, F. (2017). Enhancing cognitive theory of multimedia leaning through $3 D$ animation. Sukkur, Pakistan: Sukkur University.

Costa, B. (2014). Explaining the Pepper's ghost illusion with ray optics. Retrieved January 15, 2019, from https://www.comsol.com/blogs/ explaining-the-peppers-ghost-illusion-with-ray-optics/

Downs, J. (2006). Romancing the stone. History Today, 56, 48-54.

Ivashov, S., Capineri, L., \& Bechtel, T. (2017). Holographic subsurface radar technology and applications. In J. D. Taylor (Ed.), Ultrawideband radar: Applications and design (pp. 421-444). Boca Raton, FL; CRC Press. doi.org/10.1201/b12356

Ivashov, S., Razevig, V., Sheyko, A., Vasilyev, I., Zhuravlev, A., \& Bechtel, T. (2002). Holographic subsurface radar technique and its applications. Birmingham, UK.

Karthika, S., Praveena, P., \& GokilaMani, M. (2017). HoloLens. International Journal of Computer Science and Mobile Computing, $6,41-50$.

Knoop-van Campen, C., Segers, E., \& Verhoeven, L. (2018). The modality and redundancy effects in multimedia learning in children with dyslexia. The Netherlands: Behavioural Science Institute.

Smith, A., \& Ayres, P. (2016). Investigating the modality and redundancy effects for learners with persistent pain. Educational Psychology Review, 28, 401-424.

Uddman, C., \& Norberg, J. (2017). Augmented reality with holograms for combat management systems. Performance limitations for sonar tracks in a 3D map, presented with Microsoft HoloLens. Sweden: Degree project in electronics and computer engineering.

Voxon Photonics. (2017). About Voxon Photonics, Australia. Retrieved January 15, 2019, from https://voxon.co/\#applications-op 